\title{
Effect of Tip Profile on Atomic-Force Microscope Images: A Model Study
}

\author{
Farid F. Abraham and Inder P. Batra \\ IBM Research Division, Almaden Research Center, San Jose, California 95120 \\ and \\ S. Ciraci \\ Department of Physics, Bilkent University, Ankara, Turkey \\ (Received 8 Septemter 1987)
}

\begin{abstract}
Adopting the empirical silicon interatomic potential of Stillinger and Weber, we investigate the effect of the tip profile on the atomic-force microscope images for a prototype system, $\mathrm{Si}(001)-(2 \times 1)$, and conclude that the tip profile has a profound effect on the observations. We also study relaxation of the surface under the influence of the tip using a many-body energy minimization procedure and find that the force exerted by the tip should be less than $=10^{-9} \mathrm{~N}$ for the atomic-force microscope to be a nondestructive tool.
\end{abstract}

PACS numbers: 61.16.Di

The scanning tunneling microscope ${ }^{1}$ (STM) and more recently the atomic-force microscope ${ }^{2,3}$ (AFM) have proved to be very successful in imaging surfaces at atomic resolution. On the basis of the theory of tunneling developed by Bardeen, Tersoff and Hamann ${ }^{4}$ showed that in STM the tunneling current is proportional to the local density of states. The result becomes particularly simple for a spherical tip with an $s$-type wave function. In this case, the local density of states need only be evaluated at the center of the tip. The spherical $s$-wave tip model, for all practical purposes, acts as an isolated single-atom tip. Mizes, Park, and Harrison ${ }^{5}$ have recently considered the effect of a diatomic tip on STM images of the graphite surface and proposed an explanation for many anomalous images obtained for this system. ${ }^{6,7}$ The monoatomic-tip image of graphite is known to be the centered hexagon with $2.46-\AA$ periodicity arising ${ }^{8}$ from atoms $(B)$ having no neighbors directly below in the next lower layer.

The effect of the tip shape on the AFM images is largely unknown although it is suggested ${ }^{9}$ that even in the STM mode atomic forces play a dominant role for graphite. The inclusion of tip-surface interactions has been recently pursued, ${ }^{10-12}$ but very little is said about the effect of the tip profile on the AFM images. In this paper, we consider the effect of the tip structure and shape on the AFM images by performing force and energy calculations on a prototype $\mathrm{Si}(001)-(2 \times 1)$ surface interacting with an idealized AFM tip consisting of one to four silicon atoms. We adopt the Stillinger-Weber ${ }^{13}$ interatomic potential for calculating forces and surface relaxations (due to the presence of the AFM tip) using a molecular relaxation technique. This procedure has successfully obtained the unbuckled-dimer bond formation ${ }^{14}$ on $\mathrm{Si}(001)$ and the atomic relaxations and formation energies of self-interstitial defects in silicon. ${ }^{15}$ The Stillinger-Weber potential was optimized along an isochore for the bulk crystal and liquid, and by use of the molecular-dynamics simulation technique it was demonstrated that the interface between the two phases can be described accurately. ${ }^{16}$ The surface was modeled by an 8-layer-thick slab along the [001] direction and a vacuum space where the AFM tip was placed. To avoid end effects, the large surface unit cell (64 atoms per layer) was periodically repeated. We examined six different types of tips: $T_{1}, T_{2}, T_{2 R}, T_{3}, T_{3 R}$, and $T_{4}$ : the numerical value in the subscript gives the number of $\mathrm{Si}$ atoms constituting the particular tip. It is important to realize that currently there is no feasible formalism available for AFM at any self-consistent field level for such large sys-

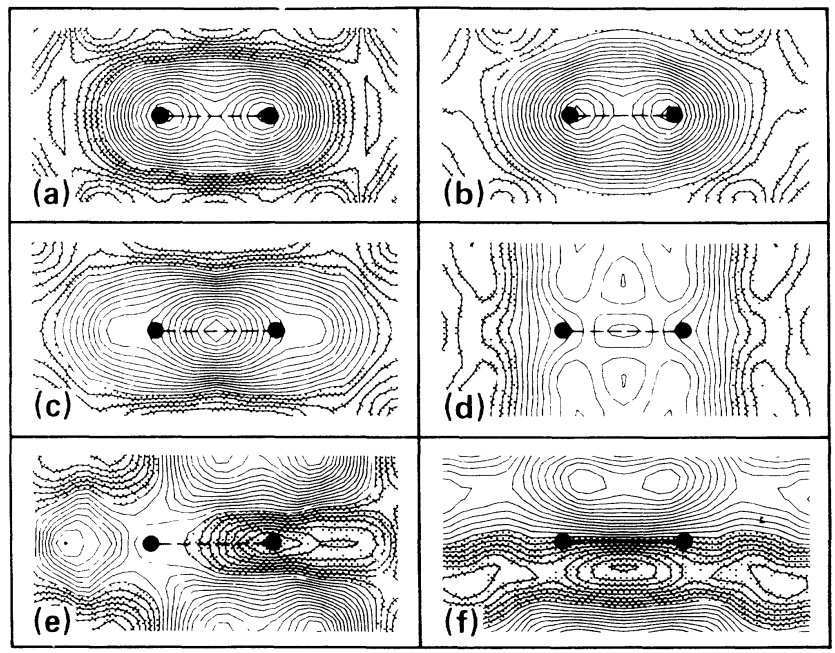

FIG. 1. The contour plots of the force on various tips above the $\mathrm{Si}(001)-(2 \times 1)$ surface, without surface relaxation: (a) $T_{1}$, (b) $T_{4}$, (c) $T_{2}$, (d) $T_{2 R}$, (e) $T_{3}$, (f) $T_{3 R}$. The shaded areas show the attractive force regions. The surface dimer atoms are indicated by filled circles. 
tems. An $a b$ initio calculation has been presented earlier ${ }^{12}$ but was restricted to a monatomic tip only. The use of empirical potentials with a many-body energy minimization procedure thus represents an important advancement for the exploration of tip geometry effects and tip-induced relaxation of the surface.

We first consider the surface and the tip atoms as rigid. We will learn later that the effect of tip-induced surface relaxation does not change the qualitative features of the analysis as long as the AFM is a nondestructive probe, i.e., when the tip is beyond $\simeq 2 \AA$ and forces are in the range of $10^{-9} \mathrm{~N}$. Figures $1(\mathrm{a})-1(\mathrm{f})$ give the contour plots for total normal forces experienced by a tip placed at a vertical distance of $\simeq 2 \AA$. To visualize the topography, the corresponding surface plots are given in Figs. 2(a)-2(f). The insets give the shapes of all the tips. There are two types of diatomic tips $\left(T_{2}, T_{2 R}\right)$ and triatomic tips $\left(T_{3}, T_{3 R}\right)$. The $T_{2}$ tip had its two atoms placed parallel to the surface dimer bonds and the $T_{2 R}$ tip had the atoms in the same $z$-plane but orthogonal to the surface dimers. Interatomic distance between the two tip atoms was set at the Si bulk bond length. The tetramer tip $\left(T_{4}\right)$ had a pyramid shape, the blunt triangular face being farthest from the surface. The triatomic tips were generated by our removing the atom at

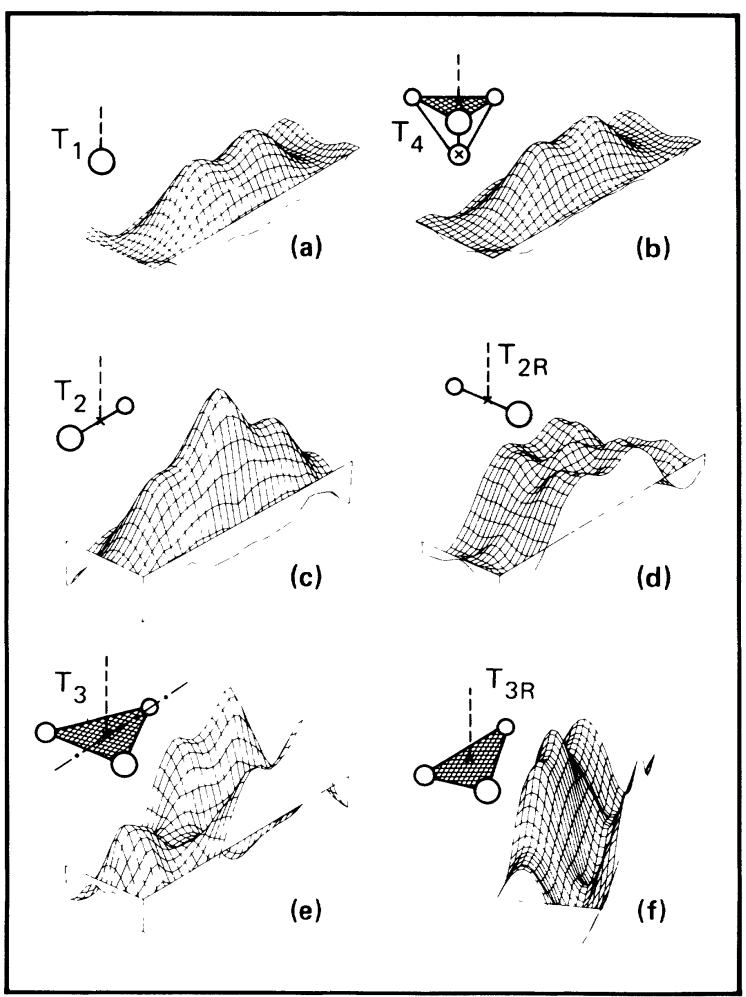

FIG. 2. The surface plots of the force on various tips above the $\mathrm{Si}(001)-(2 \times 1)$ surface, without surface relaxation: (a) $T_{1}$, (b) $T_{4}$, (c) $T_{2}$, (d) $T_{2 R}$, (e) $T_{3}$, (f) $T_{3 R}$. Height above the reference line indicates magnitude of the repulsive force. the apex. The triatomic tips were placed in two different orientations with respect to the surface. For $T_{3}$, one side of the triangle was parallel to the $y$ axis, while for $T_{3 R}$ the side was parallel to the $x$ axis (the dimer bond direction). A significant point to note is that, unlike STM where phase factors cause major complications in the study of the tip shape, the AFM offers no such difficulty. In the case that relaxation is ignored, a multiatom tip image can easily be created from a single-atom tip image by linear superposition.

From Figs. 1 and 2, one can draw conclusions about the AFM images to be seen for each tip profile. As expected, at the vertical distance of $\simeq 2 \AA$, the forces are repulsive (highlighted in Fig. 1); to date no atomic resolution has been reported in the attractive regime. We mention that only tips $T_{1}$ and $T_{4}$ resolve the surface as consisting of dimer atoms. Tip $T_{4}$ behaves much like a single-atom tip; the atoms farther from the surface do not play any significant role except for a minor distortion of the image. Tip $T_{2}$ experiences the most repulsive force when its two atoms are above the surface dimer atoms. Thus the diatomic tip shows a pronounced maximum at the center of the surface dimer atoms. Tip $T_{2}$ thus sees a single surface atom (actually the surface dimer bond). The tip $T_{2 R}$ shows a spectacular picture in that it highlights five regions in the surface cell. It experiences the most repulsive force when at least one of the tip atoms is on top of one of the surface atoms. When that happens, the center of the tip is displaced along the $\pm y$ direction from the dimer atoms, giving rise to four maxima. The fifth maximum occurs when the tip atoms bisect the surface dimer bond orthogonally. Tip atoms are equidistant from the surface atoms and this lead to a repulsive force at the tip. The tip $T_{3}$ shows an unusual picture of the unit cell [see Figs. 1(e) and

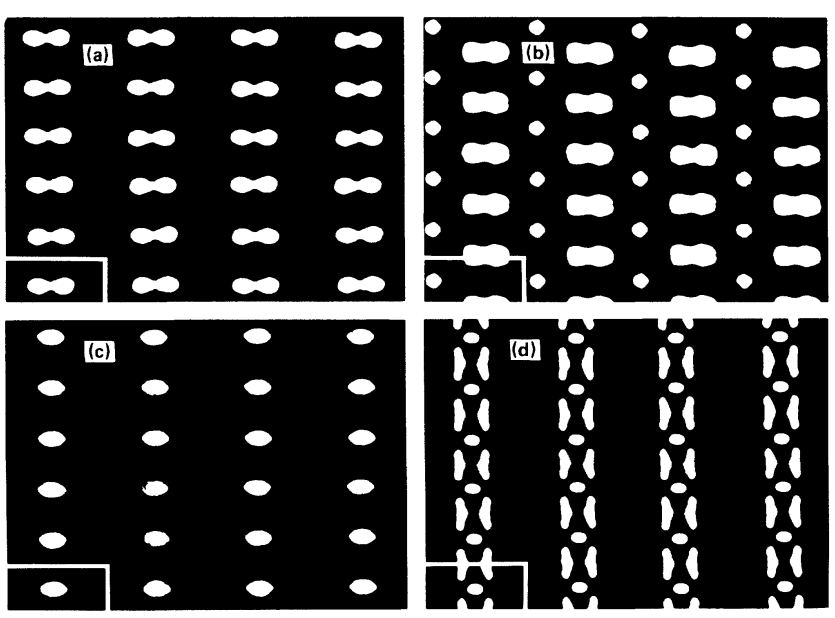

FIG. 3. Black and white AFM images of $\mathrm{Si}(001)-(2 \times 1)$ expected from four different tips and no surface relaxation: (a) $T_{1}$, (b) $T_{3}$, (c) $T_{2}$, and (d) $T_{2 R}$. 
TABLE I. Calculated normal forces (repulsive, with positive sign) on a monoatomic tip (in units of $10^{-9} \mathrm{~N}$ ) in the absence, $F_{T O}$, and in presence, $F_{T f}$, of surface relaxation for the tip in on-top $(A)$ and the mid-dimer $(M)$ sites for several heights $(h)$ above the $\mathrm{Si}(001)-(2 \times 1)$ surface.

\begin{tabular}{ccrr}
\hline \hline Site & $h(\AA)$ & \multicolumn{1}{c}{$F_{T O}$} & \multicolumn{1}{c}{$F_{T f}$} \\
\hline$A$ & 1.06 & 428.15 & 2.92 \\
$M$ & 1.06 & 63.02 & 2.03 \\
$A$ & 1.54 & 57.28 & 3.50 \\
$M$ & 1.54 & 23.02 & -0.18 \\
$A$ & 2.02 & 9.18 & 1.00 \\
$M$ & 2.02 & 6.49 & -0.75 \\
$A$ & 2.50 & -1.82 & -0.54 \\
$M$ & 2.50 & -2.40 & -1.43 \\
\hline
\end{tabular}

2(e)]. It shows the surface dimers but at the side of the unit cell rather than in the middle. In addition, it images a single atom at the left-hand side of the unit cell. Such an AFM image would lead to all kinds of faulty interpretations unless the tip shape is properly taken into account. This image can be readily understood if during the scan it is kept in mind that the short side of the unit cell is equal to the side of the tip. The image due to the tip $T_{3 R}$ can be similarly constructed. In Fig. 3 we have created black and white AFM images for several tips. A repulsive tip force above a certain critical value is shown in white and below this critical value is shown in black. White areas thus represent maximum deflection of the cantilever in the AFM. It is obvious that the true image of the surface is obtained with a monoatomic tip.

So far our discussion has assumed a rigid surface and tip. The reconstruction of the surface's atomic topography would be significant when the tip's proximity to the surface is sufficiently close. Hence, it is important to determine under what conditions the surface definition can be maintained while accounting for surface relaxation at various tip-surface separations. Our molecular relaxation calculations are uniquely suited to examine this issue. For a single-atom tip, located at various heights above $\boldsymbol{A}$ and $\boldsymbol{M}$ sites, the initial and final (after surface has fully relaxed) calculated forces on the tip are given in Table I. Note that when the tip height is less than $\approx 1.5 \AA$ from the surface, the relaxation is severe and the force topography is significantly reduced. Under these circumstances the AFM tip will alter the surface being investigated. The relaxation-induced reduction of force is still likely to reflect surface topography only when the tip is beyond $\simeq 2 \AA$ and forces in the range of $10^{-9} \mathrm{~N}$.

Figure 4 shows the contours for the force experienced by the tip after complete surface relaxation. The forces on the single-atom tip were calculated at a fine $x y$ grid for a height $\simeq 2 \AA$ of the single-atom tip above the surface. The forces are still repulsive around the surface

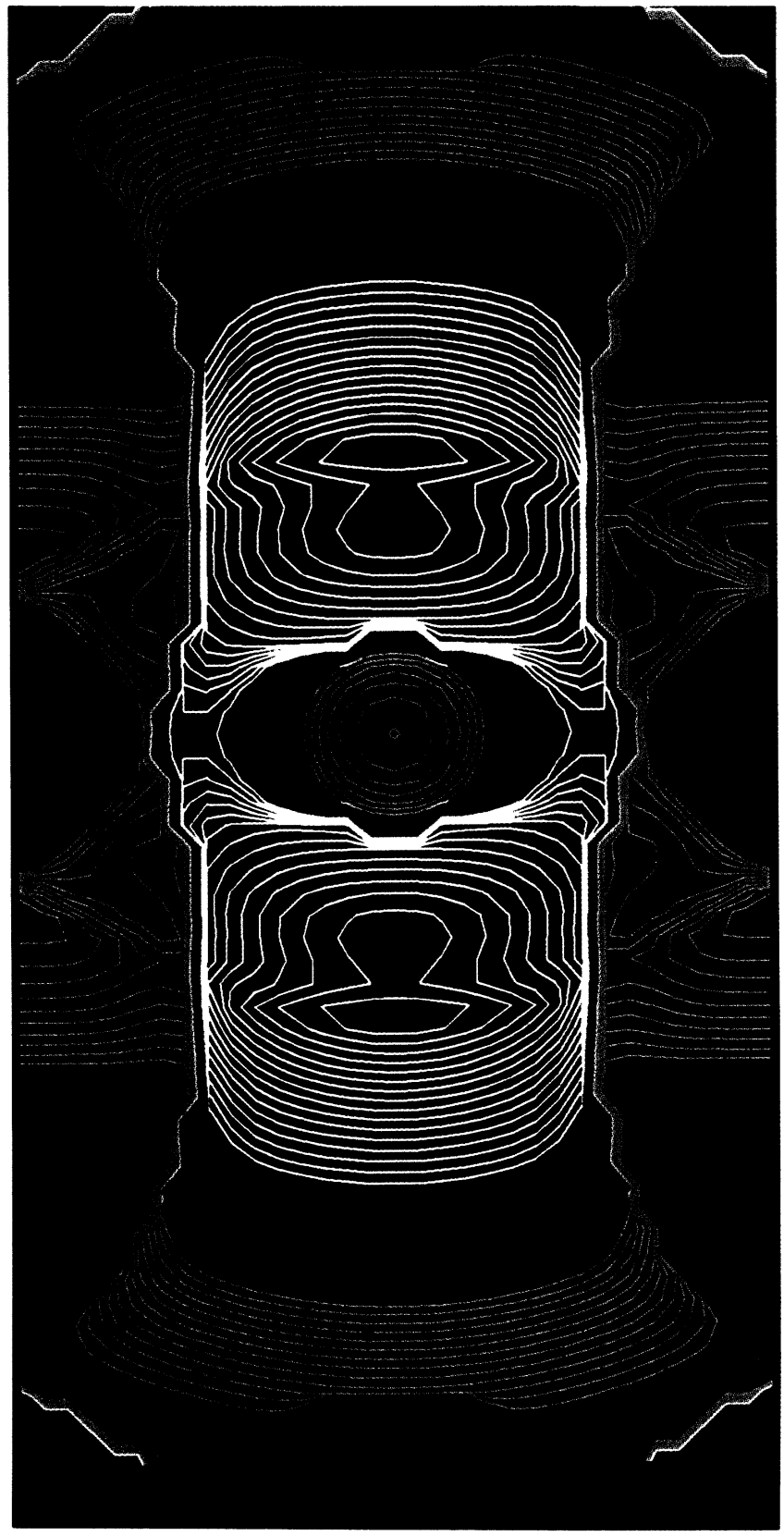

FIG. 4. The contour plots of the force experienced by the tip $T_{1}$ (held at $=2 \AA$ above the surface) after the complete relaxation of the surface due to the tip's presence at each surface $(x y)$ site. Contours in white show the repulsive regime. Attractive force in the range of 0 to -0.83 is shown in turquoise, between -0.83 and -1.66 in yellow, between -1.66 and -2.49 in green, between -2.49 and -3.32 in red, and less than -3.32 in blue. All forces are in units of $10^{-9} \mathrm{~N}$.

atoms but in between (at the dimer bond site) the force is now attractive. The attractive force indicates weakening of the dimer bond due to the presence of the tip. In the absence of surface relaxation the forces were repul- 
sive at both sites [see Table I and Fig. 1(a)]. Thus the AFM would resolve the atoms but perhaps with somewhat reduced contrast as a result of relaxation. Since the atomic relaxation occurs on a much shorter time scale as compared to the scan speed, the relaxation will occur in real systems and will tend to smooth out the force corrugation. Also, it should be noted that a multiatom tip image cannot be created from a single-atom tip image by a simple superposition if surface relaxation due to the tip's presence is allowed.

In summary, it can be stated that the tip profile can have profound effect on the AFM images. Furthermore, if the force between the tip and the surface is too repulsive, the surface atoms will significantly relax to lower forces and the resulting image may not be that of the undisturbed surface. This has important implications for studies of biological materials with use of AFM which have tremendous flexibility and can readily relax. For silicon we find that the force exerted by the tip should be less $\simeq 10^{-9} \mathrm{~N}$ for AFM to be a nondestructive tool.

${ }^{1}$ G. Binnig, H. Rohrer, Ch. Gerber, and E. Weibel, Phys. Rev. Lett. 49, 57 (1982), and 50, 120 (1983).

${ }^{2}$ G. Binnig, C. F. Quate, and Ch. Gerber, Phys. Rev. Lett. 56, 930 (1986).

${ }^{3}$ G. Binnig, Ch. Gerber, E. Stoll, T. R. Albrecht, and C. F.
Quate, Europhys. Lett. 3, 1281 (1987).

${ }^{4}$ J. Tersoff and D. R. Hamann, Phys. Rev. Lett. 50, 1998 (1983).

${ }^{5}$ H. A. Mizes, Sang-Il Park, and W. A. Harrison, Phys. Rev. B 36, 4491 (1987).

${ }^{6}$ G. Binnig, H. Fuchs, Ch. Gerber, H. Rohrer, E. Stoll, and

E. Tosatti, Europhys. Lett. 1, 31 (1985); Sang-Il Park and

C. F. Quate, Appl. Phys. Lett. 48, 112 (1986).

${ }^{7}$ J. Schneir, R. Sonnenfeld, P. K. Hansma, and J. Tersoff, Phys. Rev. B 34, 4979 (1986).

${ }^{8}$ I. P. Batra, N. Garcia, H. Rohrer, H. Salemink, E. Stoll, and S. Ciraci, Surf. Sci. 181, 126 (1987).

${ }^{9}$ J. M. Soler, A. M. Baro, N. Garcia, and H. Rohrer, Phys. Rev. Lett. 57, 444 (1986).

${ }^{10}$ U. Duerig, J. K. Gimzewski, and D. W. Pohl, Phys. Rev. Lett. 57, 2403 (1986).

${ }^{11} \mathrm{~J}$. B. Pethica, to be published; J. K. Gimzewski and R. Moller, Phys. Rev. B 36, 1284 (1987).

${ }^{12}$ I. P. Batra and S. Ciraci, in Proceedings of Second International Conference on Scanning Tunneling Microscopy/Spectroscopy, Oxnard, California, July 20-24, 1987, J. Vac. Sci. Technol. (to be published); S. Ciraci and I. P. Batra, Phys. Rev. B 36, 6194 (1987).

${ }^{13}$ F. H. Stillinger and T. A. Weber, Phys. Rev. B 31, 5262 (1985).

${ }^{14}$ F. F. Abraham and I. P. Batra, Surf. Sci. 163, L752 (1985).

${ }^{15}$ I. P. Batra, F. F. Abraham, and S. Ciraci, Phys. Rev. B 35, 9552 (1987).

${ }^{16}$ F. F. Abraham and J. Q. Broughton, Phys. Rev. Lett. 56, 734 (1986). 


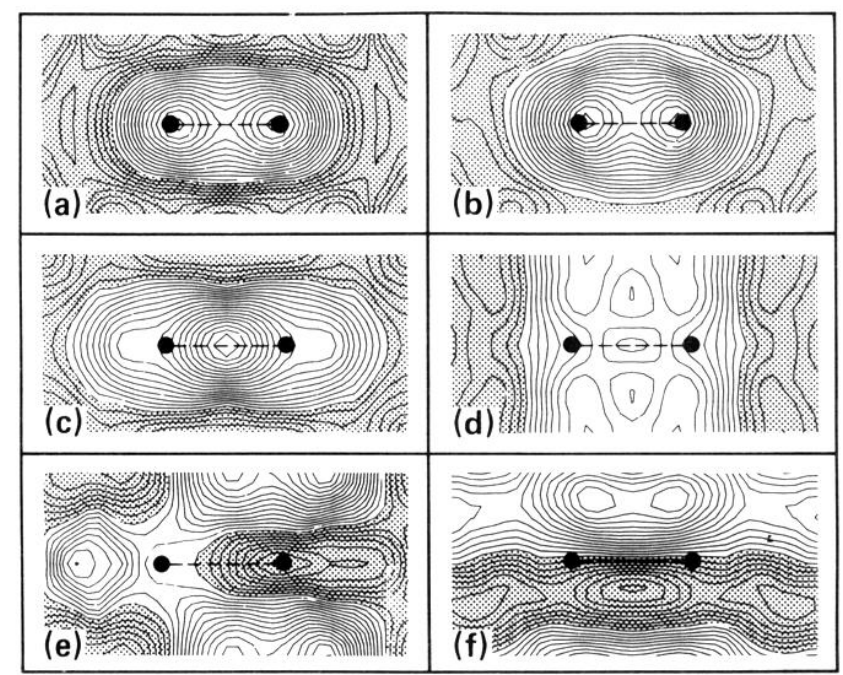

FIG. 1. The contour plots of the force on various tips above the $\mathrm{Si}(001)-(2 \times 1)$ surface, without surface relaxation: (a) $T_{1}$, (b) $T_{4}$, (c) $T_{2}$, (d) $T_{2 R}$, (e) $T_{3}$, (f) $T_{3 R}$. The shaded areas show the attractive force regions. The surface dimer atoms are indicated by filled circles. 

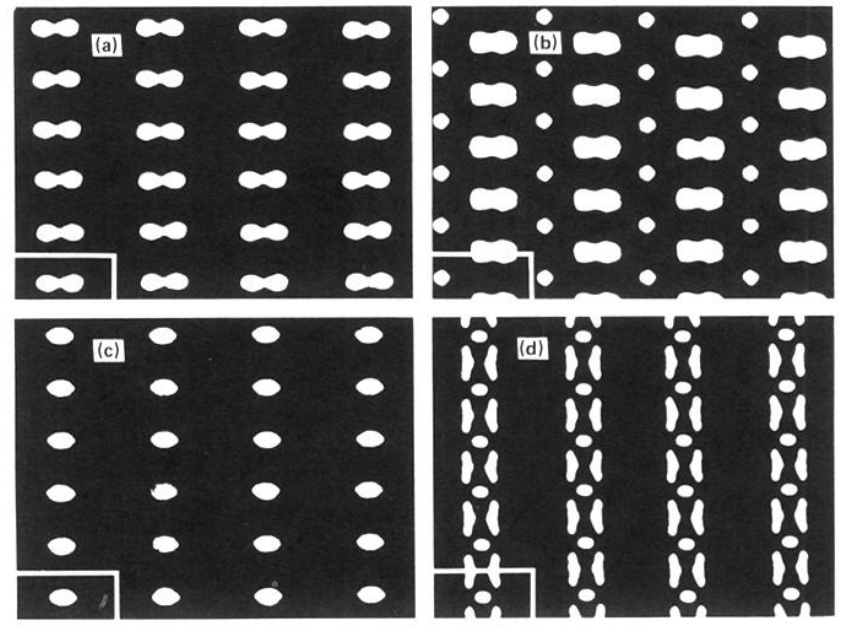

FIG. 3. Black and white AFM images of $\mathrm{Si}(001)-(2 \times 1)$ expected from four different tips and no surface relaxation: (a) $T_{1}$, (b) $T_{3}$, (c) $T_{2}$, and (d) $T_{2 R}$. 


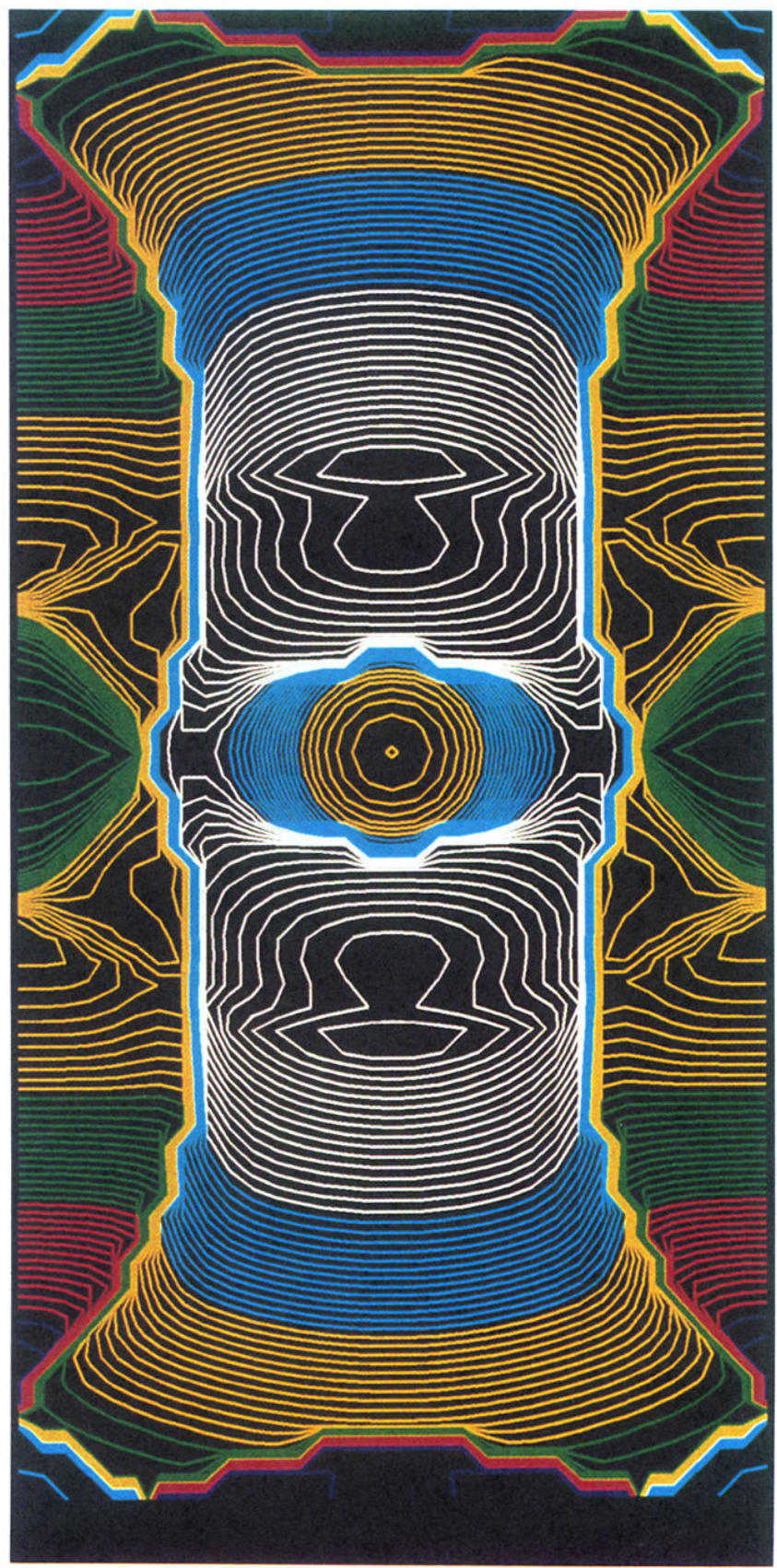

FIG. 4. The contour plots of the force experienced by the tip $T_{1}$ (held at $\simeq 2 \AA$ above the surface) after the complete relaxation of the surface due to the tip's presence at each surface (xy) site. Contours in white show the repulsive regime. Attractive force in the range of 0 to -0.83 is shown in turquoise, between -0.83 and -1.66 in yellow, between -1.66 and -2.49 in green, between -2.49 and -3.32 in red, and less than -3.32 in blue. All forces are in units of $10^{-9} \mathrm{~N}$. 